\title{
Sudden infant death. Not seeking for a culprit, but someone responsible for prevention
}

The unexpected death of an infant was already mentioned in the Old Testament's Book of Kings (Chapter 3, verse 19), which reads: "And this woman's child died in the night: for in her sleep she overlaid him." This event gave King Solomon a chance to show the greatness of his wisdom.

Maybe it is time to ask ourselves how much we know about this subject but, most importantly, how to apply such knowledge. Not knowing is unfortunate, but knowing and not acting in accordance with such knowledge is much more serious.

Sudden infant death has always existed, but it was only defined with a proper name in 1969, when a small group of doctors and scientists, gathered on an island in North Puget Sound (USA), named these unexplained deaths "sudden infant death syndrome" (SIDS).

We know that not every unexpected death is a sudden infant death. Sudden death is, by definition, a death occurring in an infant for which a cause cannot be assigned after analyzing the child's medical record, performing a complete autopsy and evaluating the place where the incident occurred.

Of course, deaths caused by intentional killing (infanticide) and non intentional deaths caused by accidents and suffocation do not fall into this definition. Even though their differences may appear clear, they are not because every human action that results in harm to another individual will always raise suspicion regarding its intentionality, either by action or omission. The death of a young child has always led to think that there is someone to blame. Either because it was intentional or because someone did not adequately comply with his/her caregiver role.

A case that occurred in Connecticut in 1958 led the child's parents, Mr. and Mrs. Roe, to create the National Sudden Infant Death Syndrome Foundation for the promotion of research regarding this confusing subject.

In 1971, the National Institute of Child Health and Human Development convened the first conference on SIDS research. International meetings were then held periodically to discuss research advances.

The first prevention campaigns were designed in the 90s; they were initially carried out in Australia, New Zealand, the USA and some Euro- pean countries, and then were spread around the world. In Argentina, the first campaign took place in 1997.

One of the characteristics of these campaigns is the change in terms of greater knowledge. The first campaigns were based on observations. However, current campaigns are based on scientific evidence. Given that sudden death and death by suffocation have many features in common, the latest recommendations by the Task Force on Sudden Infant Death Syndrome have comprehensively focused on providing infants a safe sleeping environment.

It is worth pointing out that all these subjects are thoroughly described; the scientific basis for each statement is analyzed. Subjects include sleeping position, type of surface where the child sleeps, co-sleeping, bedding, use of sleep positioners, exposure to tobacco and alcohol, breast-feeding, use of pacifiers, environment temperature, vaccine administration, education campaigns and, lastly, indications to the media and manufactures of objects related to infant sleeping, to follow the recommendations made on sudden death prevention, in all forms of dissemination.

Here, at the editorial group of Archivos Argentinos de Pediatría, a few months ago we considered writing an article on deaths by suffocation in association with the use of sleep positioners. It is published in this issue (page 62) and is aimed at warning pediatricians about the risk they imply and the situation in Argentina regarding the use of crib accessories which is against all recommendations made for preventing sudden infant death.

The Centers for Disease Control and Prevention (CDCs) of the USA have recently $(11 / 23 / 2012)$ published a report on death by suffocation related to sleep positioners in the USA between 1997 and 2011. The report points out that these kinds of death have increased four times over this period, and many are related to an unsafe sleeping environment. There are certain crib accessories approved by the USA Food and Drug Administration (FDA) to be used in case of gastroesophageal reflux and plagiocephaly, but there are also many unauthorized sleep positioners marketed for the prevention of SIDS or for more comfort during sleep. In spite of the efforts made to understand and support each action aimed at SIDS prevention, health care providers, families 
and the industry in the USA appear to follow different pathways. The situation in Argentina is not better.

The fact is that the culture related to medical errors has to be modified, as well as the cultural beliefs about sudden death. People used to look for a culprit, someone to put the blame on for such unfortunate event. Now people believe errors reflect the system failure to prevent them.

Sudden death is not absolutely avoidable. The triple-risk model explains that SIDS occurs at the confluence of three factors: a vulnerable infant, at a specific moment of life, exposed to environmental adverse conditions. We may try to reduce the birth of vulnerable children promoting pregnancy controls, avoiding exposure of pregnant women to tobacco smoke or alcohol, and preventing preterm births. The first year of life is an unchangeable risk.

We could certainly create a safe sleeping environment, and this is what we learn from prevention campaigns. There is scientific evidence indicating that the implementation of advised measures has enabled to achieve a reduction in sudden death to one third of the values prior to campaigns enforcement.

Several questions are posed: Why do people not comply with recommendations? Why ignore them? Who is responsible for their dissemination? Who is responsible for the enforcement of recommendations? Who is responsible for supervising the manufacture and sale of products that go against the recommendations?

Someone should be accountable for all these aspects, either public health authorities or intermediary institutions.

The call is open.

Norma E. Rossato Associate Editor 